\title{
DERIVATIONS OF PRIME AND SEMIPRIME RINGS
}

\author{
Nurcan Argaç and Hulya G. Inceboz
}

\begin{abstract}
Let $R$ be a prime ring, $I$ a nonzero ideal of $R, d$ a derivation of $R$ and $n$ a fixed positive integer. (i) If $(d(x) y+x d(y)+d(y) x+y d(x))^{n}=$ $x y+y x$ for all $x, y \in I$, then $R$ is commutative. (ii) If $\operatorname{char} R \neq 2$ and $(d(x) y+x d(y)+d(y) x+y d(x))^{n}-(x y+y x)$ is central for all $x, y \in I$, then $R$ is commutative. We also examine the case where $R$ is a semiprime ring.
\end{abstract}

\section{Introduction}

Throughout the paper $R$ will represent an associative ring with center $Z(R)$. For any $x, y \in R$, the symbol $[x, y]$ stands for the commutator $x y-y x$. Recall that a ring $R$ is prime if $x R y=0$ implies either $x=0$ or $y=0$, and $R$ is semiprime if $x R x=0$ implies $x=0$. An additive mapping $d: R \rightarrow R$ is called a derivation if $d(x y)=d(x) y+x d(y)$ holds for all $x, y \in R$.

In [1], M. Ashraf and N. Rehman proved that if $R$ is a prime ring, $I$ is a nonzero ideal of $R$ and $d$ is a derivation of $R$ such that $d(x) y+x d(y)+$ $d(y) x+y d(x)=x y+y x$ for all $x, y \in I$, then $R$ is commutative. In this paper we shall generalize this result, assuming that $n$ is a fixed positive integer and $(d(x y+y x))^{n}-(x y+y x)$ is 0 for all $x, y \in I$ or is central for all $x, y \in I$. We obtain some analogous results for semiprime rings in the case $I=R$.

\section{Derivations in prime rings}

In all that follows, unless stated otherwise, $R$ will be a prime ring, $I$ a nonzero ideal of $R$. For any ring $S, Z(S)$ will denote its center.

We will also make frequent use of the following result due to Kharchenko [9] (see also [12]):

Let $R$ be a prime ring, $d$ a nonzero derivation of $R$ and $I$ a nonzero twosided ideal of $R$. Let $f\left(x_{1}, \ldots, x_{n}, d\left(x_{1}\right), \ldots, d\left(x_{n}\right)\right)$ be a differential identity in $I$, that is,

$$
f\left(r_{1}, \ldots, r_{n}, d\left(r_{1}\right), \ldots, d\left(r_{n}\right)\right)=0 \text { for all } r_{1}, \ldots, r_{n} \in I .
$$

Received November 28, 2007; Revised June 20, 2008.

2000 Mathematics Subject Classification. Primary 16N60; Secondary 16W25.

Key words and phrases. prime and semiprime rings, left Utumi quotient rings, differential identities, derivations. 
One of the following holds:

1) Either $d$ is an inner derivation in $Q$, the Martindale quotient ring of $R$, in the sense that there exists $q \in Q$ such that $d(x)=[q, x]$ for all $x \in R$, and $I$ satisfies the generalized polynomial identity

$$
f\left(r_{1}, \ldots, r_{n},\left[q, r_{1}\right], \ldots,\left[q, r_{n}\right]\right)=0 ;
$$

or

2) $I$ satisfies the generalized polynomial identity

$$
f\left(x_{1}, \ldots, x_{n}, y_{1}, \ldots, y_{n}\right)=0 .
$$

Theorem 1. Let $R$ be a prime ring and $I$ a nonzero ideal of $R$, and let $n$ be a fixed positive integer. If $R$ admits a derivation $d$ such that $(d(x) y+x d(y)+$ $d(y) x+y d(x))^{n}=x y+y x$ for all $x, y \in I$, then $R$ is commutative.

Proof. If $d=0$, then $x y+y x=0$ for all $x, y \in I$. Replacing $y$ by $y z$ and using the fact that $x y=-y x$, we find that $y[x, z]=0$ for all $x, y, z \in I$ and hence $\operatorname{IR}[x, z]=0$ for all $x, z \in I$. Since $I \neq 0$ and $R$ is prime, we get $[x, z]=0$ for all $x, z \in I$, hence $R$ is commutative.

Now we assume that $d \neq 0$ and $(d(x) y+x d(y)+d(y) x+y d(x))^{n}=x y+y x$ for all $x, y \in I$. This condition is a differential identity satisfied by $I$. By using Kharchenko's theorem [9], either $d=\operatorname{ad}(A)$ is the inner derivation induced by an element $A \in Q$, the Martindale quotient $\operatorname{ring} R$, or $I$ satisfies the polynomial identity

$$
(z y+x w+w x+y z)^{n}=x y+y x \text { for all } x, y \in I .
$$

In the latter case set $z=w=0$ to obtain the identity $x y+y x=0$ for all $x, y \in I$. Then $R$ is commutative as we have just seen. Assume now that $d=\operatorname{ad}(A)$. Then $([A, x] y+x[A, y]+[A, y] x+y[A, x])^{n}=x y+y x$ for any $x, y \in I$. Since by [3] $I$ and $Q$ satisfy the same generalized polynomial identities, we have $([A, x] y+x[A, y]+[A, y] x+y[A, x])^{n}=x y+y x$ for any $x, y \in Q$. Moreover, since $Q$ remains prime by the primeness of $R$, replacing $R$ by $Q$ we may assume that $A \in R$ and $C$ is the just the center of $R$. Note that $R$ is a centrally closed prime $C$-algebra in the present situation [5], i.e., $R C=R$. By Martindale's theorem in [13], $R C$ (and so $R$ ) is a primitive ring. Since $R$ is primitive, there exists a vector space $V$ and a division ring $D$ such that $R$ is a dense ring of $D$-linear transformations over $V$.

Assume first that $\operatorname{dim}_{D} V \geq 3$.

Our aim is to show that for any $v \in V, v$ and $A v$ are linearly $D$-dependent. If $A v=0$, then $\{v, A v\}$ is $D$-dependent. So we may suppose that $A v \neq 0$. If $v$ and $A v$ are $D$-independent, since $\operatorname{dim}_{D} V \geq 3$, there exists $w \in V$ such that $v, A v, w$ are also linearly independent. By the density of $R$, there exist $x, y \in R$ such that:

$$
x v=0, \quad x A v=w, \quad y v=0, \quad y A v=0, \quad y w=v .
$$

Hence we get $(-1)^{n} v=([A, x] y+x[A, y]+[A, y] x+y[A, x])^{n} v=(x y+y x) v=0$, a contradiction. So $v$ and $A v$ are linearly $D$-dependent for all $v \in V$. Now we 
want to show that there exists $\lambda \in D$ such that $A v=\lambda v$ for any $v \in V$. Now choose $v, w \in V$ linearly independent. Since $\operatorname{dim}_{D} V \geq 3$, there exists $u \in V$ such that $v, w, u$ are linearly independent. Then $\lambda_{v}, \lambda_{w}, \lambda_{u} \in D$ such that

$$
A v=\lambda_{v} v, \quad A w=\lambda_{w} w, \quad A u=\lambda_{u} u,
$$

that is,

$$
A(v+w+u)=v \lambda_{v}+w \lambda_{w}+u \lambda_{u} .
$$

Moreover $A(v+w+u)=\lambda_{v+w+u}(v+w+u)$ for a suitable $\lambda_{v+w+u} \in D$. Then we have $0=\left(\lambda_{v+w+u}-\lambda_{v}\right) v+\left(\lambda_{v+w+u}-\lambda_{w}\right) w+\left(\lambda_{v+w+u}-\lambda_{u}\right) u$. Since $v, w, u$ are linearly independent we get $\lambda_{v}=\lambda_{w}=\lambda_{u}=\lambda_{v+w+u}$, that is, $\lambda$ does not depend on the choice of $v$. So there exists $\lambda \in D$ such that $A v=\lambda v$ for all $v \in V$.

Now for any $r \in R, v \in V$ we get $A v=v \lambda, r(A v)=r(v \lambda)$ and also $A(r v)=$ $(r v) \lambda$. Thus $0=[A, r] v$ for any $v \in V$, that is, $[A, r] V=0$. Since $V$ is a left faithful irreducible $R$-module, $[A, r]=0$ for all $r \in R$, i.e., $A \in Z(R)$ and $d=0$, which contradicts our hypothesis.

Therefore $\operatorname{dim}_{D} V$ must be $\leq 2$. In this case $R$ is a simple GPI ring with 1 , and so it is a central simple algebra finite dimensional over its center. From Lemma 2 in [11] it is clear that there exists a suitable field $F$ such that $R \subseteq$ $M_{k}(F)$, the ring of all $k \times k$ matrices over $F$, and moreover $M_{k}(F)$ satisfies the same generalized polynomial identity as $R$.

If we assume $k \geq 3$, by the same argument as in the above, we get a contradiction.

If $k=1$, then it is clear that $R$ is commutative. Thus we may assume $R \subseteq$ $M_{2}(F)$, where $M_{2}(F)$ satisfies the generalized polynomial identity ([A,x]y+ $x[A, y]+[A, y] x+y[A, x])^{n}=x y+y x$.

Let us denote $[A, x] y+x[A, y]+[A, y] x+y[A, x]$ by $K$. If we choose $x=$ $e_{12}, y=e_{21}$, then $K=0$. Hence we get $0=K^{n}=x y+y x=e_{11}+e_{22} \neq 0$, a contradiction. Therefore $k=1$, i.e., $R$ is commutative.

Lemma 1. Let $R=M_{s}(F)$, the ring of $s \times s$ matrices over a field $F$ of characteristic $\neq 2, n$ a fixed positive integer. If there exists a nonzero matrix $A$ in $R$ such that $([A, x] y+x[A, y]+[A, y] x+y[A, x])^{n}-(x y+y x) \in F$ for any $x, y \in R$, then $A$ is central.

Proof. Assume that $s \geq 3$. Let $i, j, r$ be distinct indices and $A=\sum a_{m n} e_{m n}$, with $a_{m n} \in F$. Suppose that $A$ is not diagonal. Let $a_{i j} \neq 0$ for fixed $i \neq j$. If we choose $x=e_{j r}, y=e_{r i}$ with $i, j, r$ distinct indices, then $x y+y x=e_{j i}$. Let us denote $[A, x] y+x[A, y]+[A, y] x+y[A, x]=[A, x y+y x]$ by $K$. Then

$$
K=A e_{j i}-e_{j i} A
$$

and

$$
K^{n}=\sum_{l+t=n}(-1)^{l}\left(e_{j i} A\right)^{l}\left(A e_{j i}\right)^{t}
$$


By the hypothesis we have

$$
\sum_{l+t=n}(-1)^{l}\left(e_{j i} A\right)^{l}\left(A e_{j i}\right)^{t}-\left(e_{j i}\right) \in F .
$$

All the entries of this matrix are

- $(j, i)$ entries and we don't care about them;

- the entries from the terms $\left(A e_{j i}\right)^{n}$ and $\left(e_{j i} A\right)^{n}$.

In particular from $\left(A e_{j i}\right)^{n}$, consider all the entries $a_{h j} a_{i j}^{n-1} e_{h i}$ for any $h \neq j, i$. First of all notice that these entries don't occur in $\left(e_{j i} A\right)^{n}$. Since $h \neq i$ and $(M)$ must be central, it follows that $a_{h j} a_{i j}^{n-1}=0$ for any $h \neq j, i$.

Since $a_{i j} \neq 0$, we obtain $a_{h j}=0$ for all $h \neq i, j$. Now, if we choose $x y+y x=e_{j k}$ for a fixed $k \neq i, j$, we have that

$$
\sum_{l+t=n}(-1)^{l}\left(e_{j k} A\right)^{l}\left(A e_{j k}\right)^{t}-\left(e_{j k}\right) \in F .
$$

But we know that $a_{k j}=0$ for all $k \neq i, j$, that is, the matrix $\left(M^{\prime}\right)$ is reduced to $e_{j k} \in F$, a contradiction. Therefore $a_{i j}=0$ for all $i \neq j$, i.e., $A$ is diagonal.

Now we suppose that $s=2$. Then we have

$$
[A, x y+y x]^{n}-(x y+y x) \in F \text { and } R=M_{2}(F) .
$$

If $n=1$, then $K-(x y+y x) \in F$. Choose $x=e_{11}, y=e_{12}$ so that $x y+y x=e_{12}$. Hence $B=\left[A, e_{12}\right]-e_{12} \in F$. In the matrix $B=\sum_{i, j} b_{i j} e_{i j}$ we must have $b_{11}=b_{22}$. Moreover one can see that $b_{11}=-a_{21}$ and $b_{22}=a_{21}$. Then we have $2 a_{21}=0$. Since $\operatorname{char} R \neq 2$, we get $a_{21}=0$. Similarly we can see that $a_{12}=0$. Therefore $A$ is a diagonal matrix.

Let $n=2$ and $K=\left(\begin{array}{ll}k_{11} & k_{12} \\ k_{21} & k_{22}\end{array}\right)$. So we get

$$
K^{2}=\left(\begin{array}{cc}
k_{11}^{2}+k_{12} k_{21} & k_{11} k_{12}+k_{12} k_{22} \\
k_{21} k_{11}+k_{22} k_{21} & k_{21} k_{12}+k_{22}^{2}
\end{array}\right) .
$$

Moreover since $K=[A, x y+y x]$ we get $\operatorname{trace}(K)=0$, that is, $k_{11}+k_{22}=0$. By using the this fact, we have

$$
K^{2}=\left(\begin{array}{cc}
k_{11}^{2}+k_{12} k_{21} & 0 \\
0 & k_{21} k_{12}+k_{11}^{2}
\end{array}\right) \text { and hence } K^{2} \in F .
$$

Therefore when $n$ is even, we have $K^{n} \in F$. So we get $x y+y x \in F$. Choose $x=e_{11}, y=e_{12}$, so that $x y+y x=e_{12} \in F$, a contradiction.

If $n=2 t+1$ is odd, then for any $x, y \in R$, there exists $\gamma \in F$ such that

$$
[A, x y+y x]^{2 t}[A, x y+y x]=(x y+y x)+\gamma .
$$

Moreover there exists $\beta \in F$ such that $[A, x y+y x]^{2 t}=\beta$, say

$$
\beta[A, x y+y x]=(x y+y x)+\gamma .
$$

In particular pick $x=e_{11}, y=e_{12}$, then there exist $\beta, \gamma \in F$ such that

$$
\beta\left[A, e_{12}\right]=e_{12}+\gamma .
$$


If $\beta=0$, then $e_{12} \in F$-a contradiction. Hence it follows that $\beta \neq 0$ and we have

$$
B=\beta\left[A, e_{12}\right]-e_{12} \in F .
$$

In the matrix $B=\sum_{i, j} b_{i j} e_{i j}$ we must have $b_{11}=b_{22}$. Moreover one can see that:

$$
-\beta a_{21}=b_{11} \text { and } b_{22}=\beta a_{21} \text {. }
$$

Then we have $2 a_{21}=0$. Since $\operatorname{char} F \neq 2$, we get $a_{21}=0$. Similarly we can see that $a_{12}=0$. Therefore $A$ is a diagonal matrix in any case, unless $\mathrm{s}=1$ and $R$ is commutative.

For any $\varphi$ the inner automorphism on $M_{k}(F)$, we have $[\varphi(A), \varphi(x) \varphi(y)+$ $\varphi(y) \varphi(x)]^{n}-(\varphi(x) \varphi(y)+\varphi(y) \varphi(x)) \in F$ for all $x, y \in F$ and so, by the previous case, $\varphi(A)$ must be a diagonal matrix in $M_{2}(F)$. In particular, if $\varphi(x)=$ $\left(1-e_{i j}\right) x\left(1+e_{i j}\right)$ for $i \neq j$, then $\varphi(A)=\sum_{t} a_{t t} e_{t t}+\left(a_{i i}-a_{j j}\right) e_{i j}$ must be diagonal, that is $a_{i i}=a_{j j}$ for $i \neq j$. Hence $A$ is a central matrix.

Theorem 2. Let $R$ be a prime ring with $\operatorname{char} R \neq 2, I$ a nonzero ideal of $R$ and $n$ a fixed positive integer. If $R$ admits a derivation $d$ such that $(d(x) y+x d(y)+$ $d(y) x+y d(x))^{n}-(x y+y x) \in Z(R)$ for all $x, y \in I$, then $R$ is commutative.

Proof. If $d=0$, then $x y+y x \in Z(R)$ for all $x, y \in I$ and $R$ satisfies the same identities. In this case the identity is polynomial so that there exists a field $F$ such that $R$ and $F_{n}$ satisfy the same identities. Thus pick $x=e_{12}$, and $y=e_{22}$ and $x y+y x=e_{12} \notin Z(R)$, a contradiction. Therefore $n=1$ and $R$ is commutative. We may assume that $d \neq 0$.

If $(d(x) y+x d(y))+d(y) x+y d(x))^{n}=x y+y x$ for all $x, y \in I$, then $R$ is commutative by Theorem 1 . Otherwise we have $I \cap Z(R) \neq 0$ by our assumptions. Let now $J$ be a nonzero two-sided ideal of $R_{Z}$, the ring of the central quotients of $R$. Since $J \cap R$ is an ideal of $R$, then $J \cap R \cap Z(R) \neq 0$. Hence that is $J$ contains an invertible element in $R_{Z}$, and so $R_{Z}$ is simple with 1 . By the hypothesis for any $x, y \in I$ and $r \in R$, thus $I$ satisfies the differential identity

$$
\left[(d(x) y+x d(y)+d(y) x+y d(x))^{n}-(x y+y x), r\right]=0 .
$$

If $d$ is not inner, then $I$ satisfies the polynomial identity

$$
\left[(z y+x w+y z+w x)^{n}-(x y+y x), r\right]=0
$$

by Kharchenko's theorem; and setting $z=w=0$ yields the identity $[x y+$ $y x, r]=0$. In this case it is well known that there exists a field $F$ such that $R$ and $F_{m}$ satisfy the same polynomial identities. Thus $x y+y x$ is central in $F_{m}$. Suppose $m \geq 2$ and choose $x=e_{12}, y=e_{22}$. Then $x y+y x=e_{12} \notin Z(R)$ contrary to our assumptions. This forces $m \leq 1$, i.e., $R$ is commutative.

Now let $d$ be an inner derivation induced by an element $A \in Q$. Since $d \neq 0$ we may assume that $0 \neq A$. By localizing $R$ at $Z(R)$ it is easy to see that $([A, x] y+x[A, y]+[A, y] x+y[A, x])^{n}-(x y+y x) \in Z\left(R_{Z}\right)$ for any $x, y \in R_{Z}$. Since $R$ and $R_{Z}$ satisfy the same polynomial identities, in order to prove that $R$ satisfies $s_{4}\left(x_{1}, x_{2}, x_{3}, x_{4}\right)$, we may assume that $R$ is simple 
with 1. In this case, $([A, x] y+x[A, y]+[A, y] x+y[A, x])^{n}-(x y+y x) \in Z(R)$ for all $x, y \in R$. Therefore $R$ satisfies a generalized polynomial identity and it is simple with 1 , which implies that $Q=R C=R$ and $R$ has a minimal right ideal. Thus $A \in R=Q$ and $R$ is simple Artinian; that is, $R=D_{k}$, where $D$ is a division ring finite dimensional over $Z(R)$ by [13]. Then it follows that there exists a suitable field $F$ such that $R \subseteq M_{k}(F)$, the ring of all $k \times k$ matrices over $F$, and moreover $M_{k}(F)$ satisfies the generalized polynomial identity $\left[([A, x] y+x[A, y]+[A, y] x+y[A, x])^{n}-(x y+y x), r\right]=0$ by $[11$, Lemma 2]. By Lemma $1, R$ is commutative.

Corollary 1. Let $R$ be a prime ring, $I$ a nonzero ideal of $R$ and $d$ a derivation of $R$.

(i) If $d(x) x+x d(x)=x^{2}$ for all $x \in I$, then $R$ is commutative.

(ii) If $\operatorname{char} R \neq 2$ and $d(x) x+x d(x)-x^{2} \in Z(R)$ for all $x \in I$, then $R$ is commutative.

Proof. (i) Linearizing $d(x) x+x d(x)=x^{2}$ for all $x \in I$ we get $d(x) y+x d(y)+$ $d(y) x+y d(x)=x y+y x$ for all $x, y \in I$. Now apply Theorem 1 for $n=1$.

Similarly (ii) can be proved by using Theorem 2 .

The following examples show that we cannot omit the primeness condition on Theorem 1.

Example. Let $S$ be any commutative ring.

(i) Let $R=\left\{\left(\begin{array}{ll}a & b \\ 0 & 0\end{array}\right): a, b \in S\right\}$ and $I=\left\{\left(\begin{array}{ll}0 & a \\ 0 & 0\end{array}\right): a \in S\right\}$.

Define $d: R \rightarrow R$ by $d\left(\left(\begin{array}{ll}a & b \\ 0 & 0\end{array}\right)\right)=\left(\begin{array}{ll}0 & a \\ 0 & 0\end{array}\right)$. Then $R$ is a ring under the usual operations. It is easy to see that $I$ is a nonzero ideal of $R$ and $d$ is a nonzero derivation of $R$ such that for all positive integers $n(d(x) y+x d(y)+d(y) x+$ $y d(x))^{n}=x y+y x$ for all $x, y \in I$ but $R$ is not commutative.

(ii) Let $R=\left\{\left(\begin{array}{cc}a & b \\ 0 & c\end{array}\right): a, b, c \in S\right\}$ and $I=\left\{\left(\begin{array}{ll}0 & a \\ 0 & 0\end{array}\right): a \in S\right\}$.

Define $d: R \rightarrow R$ by $d\left(\left(\begin{array}{ll}a & b \\ 0 & c\end{array}\right)\right)=\left(\begin{array}{cc}0 & a-b-c \\ 0 & 0\end{array}\right)$. Then $R$ is a ring under the usual operations. It is easy to see that $I$ is a nonzero ideal of $R$ and $d$ is a nonzero derivation of $R$ such that $d(x) y+x d(y)+d(y) x+y d(x)=x y+y x$ for all $x, y \in I$ but $R$ is not commutative.

\section{Derivations in semiprime rings}

In all that follows, $R$ will be a semiprime ring. We will make use of the left Utumi quotient ring $U$ of $R$. So we need to mention that the definition, the axiomatic formulation and the properties of this quotient ring can be found in [2], [6], [10].

In order to prove that the same results are also valid for a semiprime ring $R$ rather than any nonzero ideal of $R$, we will make use of the following facts: 
Claim 1 ([2, Proposition 2.5.1]). Any derivation of a semiprime ring $R$ can be uniquely extended to a derivation of its left Utumi quotient ring $U$ and so any derivation of $R$ can be defined on the whole $U$.

Claim 2 ([2, p. 38]). If $R$ is a semiprime ring, then so is its left Utumi quotient ring. The extended centroid $C$ of a semiprime ring coincides with the center of its left Utumi quotient ring.

Claim 3 ([2, p. 42]). Let $B$ be the set of all the idempotents in $C$, the extended centroid of $R$. Assume $R$ is a $B$-algebra which is orthogonal complete. For any maximal ideal $P$ of $B, P R$ forms a minimal prime ideal of $R$, which is invariant under any derivation of $R$.

Theorem 3. Let $R$ be a semiprime ring and $n$ a fixed positive integer. If $R$ admits a derivation $d$ such that $(d(x) y+x d(y)+d(y) x+y d(x))^{n}=x y+y x$ for all $x, y \in R$, then $R$ is a commutative ring.

Proof. Since $R$ is semiprime, by Claim 2, $Z(U)=C$, the extended centroid of $R$, and, by Claim 1 , derivation $d$ can be uniquely extended on $U$. Since $U$ and $R$ satisfy the same differential identities (see [12]), then $(d(x) y+x d(y)+$ $d(y) x+y d(x))^{n}=x y+y x$ for all $x, y \in U$. Let $B$ be the complete boolean algebra of idempotents in $C$ and $M$ be any maximal ideal of $B$.

Since $U$ is a $B$-algebra which is orthogonal complete (see [12], p. 42, (2) of Fact 1 ), by Claim 3, $M U$ is a prime ideal of $U$, which is $d$-invariant. Denote $\bar{U}=U / M U$ and $\bar{d}$ the derivation induced by $d$ on $U$. Therefore $\bar{d}$ satisfies in $\bar{U}$ the same property of $d$ on $U$. In particular $\bar{U}$ is a prime ring and so, by Theorem 1, for all maximal ideals $M$ of $B$ we obtain that $[U, U] \subseteq M U$. Therefore $[U, U] \subseteq \bigcap_{M} M U=0$. In particular $R$ is commutative.

Theorem 4. Let $R$ be a 2-torsion free semiprime ring and $n$ a fixed positive integer. If $R$ admits a derivation d such that $(d(x) y+x d(y)+d(y) x+y d(x))^{n}-$ $(x y+y x) \in Z(R)$ for all $x, y \in R$, then $R$ is commutative.

Proof. By Claim 2, $Z(U)=C$, and by Claim $1, d$ can be uniquely defined on whole $U$. Since $U$ and $R$ satisfies the same differential identities $(d(x) y+x d(y)+$ $d(y) x+y d(x))^{n}-(x y+y x) \in Z(U)$ for all $x, y \in U$. Let $B$ the complete boolean algebra of idempotents in $C$ and $M$ any maximal ideal of $B$. As already pointed out in the proof of Theorem $3, U$ is a $B$-algebra which is orthogonal complete and by Claim 3, MU is a prime ideal of $U$, which is $d$-invariant. Let $\bar{d}$ be the derivation induced by $d$ on $\bar{U}=U / M U$. Since $Z(\bar{U})=(C+M U) / M U=$ $C / M U$, then $(\bar{d}(x) y+x \bar{d}(y)+\bar{d}(y) x+y \bar{d}(x))^{n}-(x y+y x) \in(C+M U) / M U$ for any $x, y \in \bar{U}$. Moreover $\bar{U}$ is a prime ring, hence we may conclude $\bar{U}$ is commutative by Theorem 2. This implies that, for any maximal ideal $M$ of $B$, we have $[U, U] \subseteq M U$. Hence $[U, U] \subseteq \bigcap_{M} M U=0$. In particular $R$ is commutative. 
Corollary 2. Let $R$ be a semiprime ring and $d$ a nonzero derivation of $R$.

(i) If $d(x) x+x d(x)=x^{2}$ for all $x \in R$, then $R$ is commutative.

(ii) If $R$ is 2-torsion free and $d(x) x+x d(x)-x^{2} \in Z(R)$ for all $x \in R$, then $R$ is commutative.

Proof. (i) Linearizing $d(x) x+x d(x)=x^{2}$ for all $x \in R$, we get $(d(x) y+x d(y)+$ $d(y) x+y d(x))^{n}-(x y+y x)=0$ for all $x, y \in R$. Then $R$ is commutative by Theorem 3.

Similarly (ii) can be proved by using Theorem 4 .

Acknowledgement. Authors would like to thank referee for his/her valuable suggestions and comments.

\section{References}

[1] M. Ashraf and N. Rehman, On commutativity of rings with derivations, Results Math. 42 (2002), no. 1-2, 3-8.

[2] K. I. Beidar, W. S. Martindale, and V. Mikhalev, Rings with Generalized Identities, Monographs and Textbooks in Pure and Applied Mathematics, 196. Marcel Dekker, Inc., New York, 1996.

[3] C. L. Chuang, GPIs having coefficients in Utumi quotient rings, Proc. Amer. Math. Soc. 103 (1988), no. 3, 723-728.

[4] - Hypercentral derivations, J. Algebra 166 (1994), no. 1, 39-71.

[5] J. S. Erickson, W. S. Martindale III, and J. M. Osborn, Prime nonassociative algebras, Pacific J. Math. 60 (1975), no. 1, 49-63.

[6] C. Faith, Lecture on Injective Modules and Quotient Rings, Lecture Notes in Mathematics, No. 49 Springer-Verlag, Berlin-New York, 1967.

[7] Y. Hirano, A. Kaya, and H. Tominaga, On a theorem of Mayne, Math. J. Okayama Univ. 25 (1983), no. 2, 125-132.

[8] N. Jacobson, PI-Algebras: An Introduction, Lecture Notes in Mathematics, Vol. 441. Springer-Verlag, Berlin-New York, 1975.

[9] V. K. Kharchenko, Differential identities of prime rings, Algebra i Logika 17 (1978), no. $2,220-238,242-243$.

[10] J. Lambek, Lecture on Rings and Modules, With an appendix by Ian G. Connell Blaisdell Publishing Co. Ginn and Co., Waltham, Mass.-Toronto, Ont.-London 1966.

[11] C. Lanski, An Engel condition with derivation, Proc. Amer. Math. Soc. 118 (1993), no. $3,731-734$

[12] T. K. Lee, Semiprime rings with differential identities, Bull. Inst. Math. Acad. Sinica 20 (1992), no. 1, 27-38.

[13] W. S. Martindale III, Prime rings satisfying a generalized polynomial identity, J. Algebra 12 (1969), 576-584.

Nurcan ArgaC

Department of Mathematics

SCIENCE FACULTY

Ege UnIVERSITy

35100, Bornova, Izmir, Turkey

E-mail address: nurcan.argac@ege.edu.tr 
Hulya G. Inceboz

Department of Mathematics

SCIENCE AND ART FACUlty

Adnan Menderes University

09010, Aydin, Turkey

E-mail address: hinceboz@adu.edu.tr 